\title{
Polysèmes
}

Revue d'études intertextuelles et intermédiales

11 | 2011

Cadres

\section{La marge en question dans la poésie de Gertrude Stein}

Isabelle Alfandary

\section{(2) OpenEdition}

Journals

Édition électronique

URL : http://journals.openedition.org/polysemes/640

DOI : $10.4000 /$ polysemes. 640

ISSN : 2496-4212

Éditeur

SAIT

Édition imprimée

Date de publication : 1 janvier 2011

Pagination : 69-86

ISSN : 0999-4203

\section{Référence électronique}

Isabelle Alfandary, «La marge en question dans la poésie de Gertrude Stein », Polysèmes [En ligne], 11 | 2011, mis en ligne le 01 mars 2015, consulté le 21 avril 2019. URL : http:// journals.openedition.org/polysemes/640 ; DOI : 10.4000/polysemes.640

Ce document a été généré automatiquement le 21 avril 2019

Polysèmes 


\title{
La marge en question dans la poésie de Gertrude Stein
}

\author{
Isabelle Alfandary
}

1 L'idée de cet article m'est venue d'une impression de lecture relative à l'interminable The Making of Americans ${ }^{1}$ (1925), roman-fleuve qui est à l'œuvre de Gertrude Stein ce que Finnegans Wake est à l'œuvre joycienne. Cette impression qui m'a accompagnée au long de ma lecture et a été l'occasion de multiples interférences, de pensées et d'affects incongrus et distraits, concerne la nature des marges, l'inscription du texte sur la page, ce que j'appellerai sa physionomie. C'est à ce détail, qu'il faudrait entendre aussi au sens pictural du terme, que je voudrais consacrer la présente étude. Car ce phénomène qui peut sembler accessoire de prime abord est l'effet d'une économie textuelle tout à fait spécifique à laquelle il permet l'accès. Se pencher sur ce non-lieu de l'écriture steinienne permet d'interroger certaines dimensions d'un texte singulièrement imprenable par ailleurs. Comment en effet aborder une œuvre comme The Making of Americans qui ne ménage que peu de place à son lecteur, qui - on ne l'a que trop dit - fait la part belle à la répétition et à la dérive du sens, si ce n'est par son bord, son bord littéral, matériel, en arpentant ses marges et en l'envisageant depuis celles-ci.

2 Ce qui saute aux yeux du lecteur chaque fois que son regard distrait dévie de la trajectoire naturelle de la ligne est l'aspect général du texte, ce que j'ai appelé à l'instant son économie, au sens que retient Littré « arrangement réciproque et concourant des parties d'un ensemble ». Ce qui pourrait se lire comme le plus long poème en prose de la modernité (925 pages) est l'effet d'un continuum de signes organisés en paragraphes d'une longueur qui ne peut pas ne pas frapper le lecteur, paragraphes qui vont parfois jusqu'à couvrir l'intégralité d'une page. Gertrude Stein n'est ni Stéphane Mallarmé, ni E.E. Cummings : ce qu'il est convenu d'appeler la «mise en page » est chez elle pour le moins rudimentaire.

3 La marge n'est pas seule à manquer : elle fait signe vers un manque à l'échelle de la page tout entière et pourquoi pas de l'œuvre, le défaut de ce que Mallarmé désigne dans le Coup de dés ${ }^{2}$ comme "surface vacante et supérieure ». Ce qui fait ironiquement défaut à The Making of Americans comme à de nombreux poèmes en prose est précisément la vacance 
des signes, la suspension de l'écriture, le blanc. Ce qui manque est une économie textuelle et typographique qui ferait une place au manque, justement. Il semble que le texte steinien ait, à l'instar de la nature, une sainte horreur du vide. Sa compacité, sa surcharge ont pour effet de saturer la lecture, de susciter une résistance. Celle-ci est au moins autant due au devenir glossolalique de la langue qu'au sentiment de saturation visuelle que dégage l'imprimé - l'un et l'autre effet étant d'ailleurs indissolublement liés. La stase à laquelle l'économie de la page steinienne oblige son lecteur est une expérience esthétique d'une rare violence qui explique les réactions de rejet dont le poète a pu faire l'objet. Le lecteur est affronté à un mur de petits signes noirs qui font inexorablement signe vers l'infini et lui signifient son impuissance à embrasser la totalité, à subsumer le divers de la lettre, à venir à bout, même temporairement, même illusoirement, de sa finitude essentielle. Le paragraphe steinien massif constitue la langue comme masse, massif de signes, obstacle à la lecture, décourageant toute lecture silencieuse et dans certains cas la lecture tout court. Le peu de marge, le manque du blanc typographique constituent le texte non seulement comme objet visuel, mais comme objet total, donc par définition impossible. La tyrannie qu'exerce le texte sur son lecteur relève d'une économie du signe que je qualifierais de rétinienne. L'œil est dans l'impasse ou bien à la dérive. Ce dernier n'en est pas moins aux premières loges d'un prodigieux spectacle textuel.

4 La pratique de l'écriture au kilomètre qui ne connaît quasiment pas de division en paragraphes, chapitres, etc. est à l'origine d'un vertige de lecture : la double page du livre habituellement invisible apparaît à la manière d'un étrange diptyque. Des désinences signifiantes - ainsi la forme dite en «-ing » dans le passage qui nous occupe - clignotent à la surface de la page, constituant une pulsation littérale inédite, à l'origine d'un plaisir de lecture très singulier; cependant l'appareil désinentiel étant relativement limité en anglais, les signes finissent immanquablement par tournoyer et s'abîmer dans un brouillard de lettres. L'intensité de l'impression tient à la densité presque sans faille d'un texte sans relief. La syntaxe steinienne travaille de concert avec sa typographie par défaut en vue d'aplanir les relations hiérarchiques entre les parties du discours et les signes. Le défaut de marge dissout l'ordre dans l'espace aussi bien que dans la langue et donne à voir l'écriture comme toile, tissu, masse, matière.

Celle qui fut une grande collectionneuse d'art moderne, l'amie des peintres que l'on sait, était dotée d'un œil très sûr. Ainsi qu'elle le confesse dans The Autobiography of Alice B. Toklas, la langue fut pour elle un objet d'expérience visuelle :

When I first knew Gertrude Stein in Paris I was surprised never to see a french book on her table, although there were always plenty of english ones, there were even no french ones. But do you never read french, I as well as many other people asked. No, she replied, you see I feel with my eyes and it does not make any difference to me what language I hear, I don't hear a language, I hear tones of voice and rhythms, but with my eyes I see words and sentences and there is for me only one language and that is english. One of the things that I have liked all these years is to be surrounded by people who know no english. It has left me more intensely alone with my eyes and my english. ${ }^{3}$

Quels sont ces mots de la langue américaine que voyait Gertrude Stein à Paris, qu'elle jetait sur le papier, qu'elle ne cessait pas de jeter sur le papier sans que ceux-ci ne trouvent nécessairement à se lire ? La frénésie scripturaire steinienne est attestée par le volume de ses écrits, et confirmée par la masse non négligeable d'inédits que recèle la collection Stein à Yale. Ces mots qui sont les mots d'une langue prétendument 
indifférente aux contingences de la syntaxe de l'anglais aussi bien qu'à la réalité matérielle de la page, marginalisent la part de la lecture, et mettent in fine le lecteur sur la touche. Si la langue qu'écrit Gertrude Stein flirte délibérément avec la langue privée, c'est qu'une inscription ininterrompue est une contradiction dans les termes, l'illimité n'ayant pas vocation à devenir un écrit. «I write for myself and for strangers » répète à l'envi le narrateur de The Making of Americans, non sans laisser son lecteur défait, interdit. Au sujet de la clinique du mélancolique, le psychanalyste Jacques Hassoun écrit :

Aussi ne peut-il susciter que des narrations sans fiction, tant il semble manquer, à l'endroit même de ses écrits qui sont pris d'un ressassement infini, de ce qui donne à l'écriture sa gravité : la marge. Car si celle-ci représente graphiquement le lieu de la respiration, le lieu où l'Autre - comme lecteur - est convoqué pour s'emparer du texte afin d'y écrire ses réflexions, ses graffitis, ses manifestations de vie, ses interprétations, alors nous pouvons concevoir le dit d'un mélancolique comme une sentence sans appel possible [...]. ${ }^{4}$

7 L'indifférence qu'affiche l'écriture steinienne peut être directement imputée au défaut de marges: la gravité dont parle Jacques Hassoun peut s'entendre dans tous les sens du terme. La gravité s'entend comme phénomène physique - le texte ne «tombant » pas comme il faudrait sur la page s'offre au regard du spectateur, devient un objet d'expérience en soi -; la gravité s'entend dans le même mouvement comme cause de l'affect. C'est comme si en l'absence de marge, le texte ne pouvait être pris tout à fait au sérieux, comme si en l'absence d'un lieu aménagé à l'attention du destinataire de l'écrit, ce dernier ne pouvait se sentir pleinement visé et donc touché par lui.

L'écriture steinienne est pratiquement sans gravité. Et pourtant, l'existence de marges, même rétrécies, et d'autres divisions, aussi minimales soient-elles, l'empêche in extremis de sombrer dans l'idiolecte. L'écriture poétique se tient au bord du précipice de la glossolalie, sur le fil du rasoir. Elle fraye aux confins de la langue, au bord d'une langue dont le jeu aurait fait place au simulacre. La langue qu'écrit Gertrude Stein peut être dite "limite » au sens où l'on parle d'états ou de cas-limite - «border line». Gertrude Stein est à la critique littéraire ce que les structures borderline sont à la nosologie psychiatrique, des « cas », des objets inclassables, impossibles et réels à la fois.

9 La physionomie du texte qui interpelle et interdit son lecteur, quoique rarement commentée dans la mesure où Gertrude Stein n'est pas connue pour ses innovations typographiques, lui confère cependant une identité visuelle remarquable. C'est en conscience qu'elle s'abstient de tout recours à la ponctuation. Si le poète qualifie la question de " vitale », c'est que de telles marques infralinguistiques n'ont à ses yeux rien d'anodin et sont au contraire susceptibles de provoquer des effets insidieux sur la conscience du lecteur et de nuire à la réception du texte.

[B]ut when I began writing, the whole question of punctuation, was a vital question. [...] The comma was just a nuisance. If you got the thing as a whole, the comma kept irritating you all along the line. If you think of a thing as a whole, and the comma keeps sticking out, it gets on your nerves; because, after all, it destroys the reality of the whole. So I got rid more and more of commas. Not because I had any prejudice against commas; but the comma was a stumbling block. When you were conceiving a sentence, the comma stopped you. That is the illustration of the question of grammar and parts of speech, as part of the daily life as we live it. ${ }^{5}$

Les difficultés qu'éprouve Gertrude Stein avec la ponctuation tiennent à la scansion. L'intuition inaugurale du poète que la ponctuation vient contrarier peut se résumer en une phrase: "When I first began writing, I felt that writing should go on ${ }^{6}$. La ponctuation freine par définition le défilé du signifiant sous la plume, ralentit le cours de 
la parole en instituant des pauses plus ou moins définitives, des inflexions plus ou moins marquées. À l'exception du point qui semble trouver grâce à ses yeux, les autres signes de ponctuation ne lui inspirent que défiance. Accessoire de la langue en écriture, la ponctuation est jugée contraire au désir steinien d'une parole sans fin et sans interférence. Loin d'être sous-estimé, le pouvoir d'impression de la ponctuation est jugé si puissant que celle-ci s'en trouve révoquée. Cet objet de préoccupation a taraudé Stein à ses débuts et a donné lieu à l'élaboration d'une véritable doctrine dont on perçoit comme toujours chez Stein ce qu'elle doit à un mécanisme de défense subjectif :

I have had a long and complicated life with all these. Let us begin with these I use the least first and these are colons and semi-colons, one might add to these commas.

When I first began writing, I felt that writing should go on, I still do feel that it should go on but when I first began writing I was completely possessed by the necessity that writing should go on and if writing should go on what had colons and semi-colons to do with it, what had commas to do with it, what had periods to do with it what had small letters and capitals to do with it to do with writing going on which was at that time the most profound need I had in connection with writing? What had colons and semi-colons to do with it what had commas to do with it what had periods to do with it?

What had periods to do with it. Inevitably no matter how completely I had to have writing go on, physically one had to again and again stop sometime and if one had to again and again stop some time then periods had to exist. Beside I had always liked the look of periods and I liked what they did. Stopping sometime did not really keep one from going on, it was nothing that interfered, it was only something that happened, and as it happened as a perfectly natural happening, I did believe in periods and I used them. I really never stopped using them. ${ }^{7}$

11 Le poète expose dans "Poetry and Grammar» une position de principe sur la ponctuation, avant de passer en revue une à une toutes les marques, de dresser comme pour les parties du discours, le catalogue interminable et raisonné de ses préférences. On le comprend, l'interruption quelle que soit sa nature, n'est pas consentie sans difficulté ni réticence. La typographie est par définition scansion, espacement, séparation, coupure. Guillaume Apollinaire justifiait d'ailleurs sa non-ponctuation dans des termes tout à fait comparables: "C'est une nouveauté. Il m'a paru que la ponctuation alourdissait singulièrement l'essor d'un poème. Celui-ci d'un seul trait, poursuit sa course ailée. Evidemment, on ne comprend pas. Mais, n'est-ce pas, cela n'a aucune importance $»^{8}$.

La marge, le blanc relèvent en partie d'une nécessité ignorée, qui tient à la ligne d'ailleurs plus qu'au vers, d'un besoin physiologique que note Gertrude Stein, celui du repos du corps de celui qui lit aussi bien que de celui qui écrit, auxquels nul ne peut se soustraire. Qu'il s'agisse pour le lecteur de reprendre son souffle ou de reposer le muscle de sa main pour l'écrivain, le corps ne se passe pas de pause, de respiration. Une telle naturalisation de la convention typographique met au jour sa raison d'être impensée. Ce que les maigres marges trahissent, ce que les espaces restreints entre les paragraphes reflètent, est l'empreinte d'un corps imaginaire, le négatif d'une image de corps sans relâche, sans respiration; et pourquoi pas sans rythme. La notion de rythme en effet repose sur l'alternance de temps forts et de temps faibles et ne se conçoit pas sans la concession d'un relâchement, le respect d'un temps mort. Le corps tel que l'écriture de Gertrude Stein le fantasme est le corps d'avant la naissance ou le corps d'après la mort, un corps hors du temps, idéal, glorieux, ignorant du besoin. Le corps du texte serait l'effet - métaphore (effet d'un déplacement) ou métonymie (effet de contiguïté) -, d'un corps littéralement à l'œuvre, impression fugace d'un corps étalé sur la page. Le corps du texte steinien n'est 
aucunement l'image au miroir du corps réel de son auteur, ce corps passé à la postérité dans des portraits qu'en firent Picasso ou Picabia, mais l'effet d'un corps imaginaire qui, même s'il a travaillé le corps réel, ne peut en aucun cas être confondu avec lui.

La seule limite que reconnaît l'écriture est la réalité de la fatigue de la main; la seule contrainte à laquelle elle consent à se soumettre est celle du support. Le texte a pour seul cadre la page du livre : le centrage qui est le sien est celui opéré in fine par l'éditeur. Pour toute borne, le texte reconnait la tranche de la feuille qui signifie la fin de la page. L'écriture postulée comme infinie n'échappe pas à l'expérience de la finitude matérielle, réelle, à l'épuisement du corps. C'est encore et toujours le corps, celui de l'écrivain ou même de la page, le corps même réduit à sa plus simple expression - l'étendue - qui en tant qu'il est marqué par le besoin ou la nécessité entrave la jouissance illimitée de la parole.

14 La consultation des manuscrits nous a permis de conforter et d'étayer notre intuition relative au déficit de marges. Gertrude Stein occupe tout l'espace de la ligne, déborde des emplacements typographiques aménagés par les cahiers. Toutes les œuvres y compris les plus volumineuses sont en effet composées sur de petits cahiers d'écolier au format invariable. L'écriture y apparaît comme relativement rare, chaque ligne ne comportant que quelques mots, chaque page ne comptant qu'exceptionnellement plus d'une poignée de lignes.

La légèreté du trait du crayon de papier dont use le plus souvent Gertrude Stein contraste avec l'absence troublante de rature ou de biffure, la régularité métronomique de l'écriture, sa détermination sans objet, son affolante tranquillité. La machine à écrire steinienne qui remplit des milliers de pages de petits cahiers sans ciller, désaffectée au point d'en devenir indifférente, est impressionnante. La manifestation la plus remarquable de ce phénomène est l'absence de la moindre trace d'affect qui semble avoir présidé à la rédaction du sulfureux Tender Buttons (1914). La page imprimée est en l'espèce en tout point fidèle à l'esprit du manuscrit: le trait steinien ne laisse pas paraître la moindre marque subjective. Le poète ne corrigeait pas plus semble-t-il le manuscrit que le tapuscrit, religieusement dactylographié par Alice B. Toklas. La main du poète n'hésite ni ne tremble; l'écrit steinien ne s'amende pas. Il est irréversible, inévitable, finalement intouchable. L'écriture ne se cherche pas, elle se trouve pour paraphraser la formule célèbre de Pablo Picasso. Elle est sans programme, ni remords.

Le temps de l'écriture, le temps avec lequel celle-ci se confond, ainsi que Gertrude Stein en a la perception très précoce dans Three Lives (1909) est le présent, ce même présent dont les formes en "-ing» de The Making of Americans se ressentent si fortement. L'écriture est saisie de l'instant, capture de la langue en tant qu'elle a lieu. L'inspiration est absolument étrangère à la poétique steinienne: la consultation des archives fait apparaître que le patronyme du personnage éponyme d'une pièce en prose «Brim Beauvais. A Novelette. 1931 » n'est autre que celle du papetier chez lequel Gertrude Stein se fournissait en précieux petits cahiers ainsi que l'atteste la couverture bleue: «Beauvais, 14 rue du Bac, Paris, Tél Littré 5487 ». Il n'y a pas d'au-delà du texte; ce dernier est tout entier dans l'ici et maintenant de l'énonciation.

De Beauvais à Brim Beauvais, il n'y a qu'un pas que franchit le poème, qui, au-delà de l'anecdote, est singulièrement parlant, notoirement significatif d'un régime métonymique à l'origine d'une poésie fondée sur des relations de contiguïté entre les signes. Le rapport de contiguïté qu'exalte la langue steinienne entre tous est le son. La musique de la langue est recherchée à travers des procédés de variations syllabiques et de 
systèmes de glissements allitératifs complexes : les relations qu'entretiennent les signes entre eux sont fondées sur la ressemblance phonématique plus que sur toute autre forme d'isotopie. Les liens logiques se distendent, se perdent au profit de liens phonétiques. Si l'on considère avec Jean Cohen, auteur de Structure du langage poétique, que ce sont les phonèmes «qui par leurs combinaisons, composent le lexique, c'est-à-dire portent les significations $"$, on prend la mesure de la violence que l'écriture steinienne fait à la langue, retournant les phonèmes contre eux-mêmes. Ainsi, cet extrait du livret d'un des deux opéras que composa Gertrude Stein : Four Saints in Three Acts (1934).

Scene II

Pigeons on the grass alas.

Pigeons on the grass alas.

Short longer grass short longer longer longer shorter yellow grass. Pigeons large pigeons on the shorter longer yellow grass alas pigeons on the grass.

If they were not pigeons what were they.

If they were not pigeons on the grass alas what were they. He had heard of a third and he asked about it it was a magpie in the sky. If a magpie in the sky on the sky can not cry if the pigeon on the grass alas can alas and to pass the pigeon on the grass alas and the magpie in the sky on the sky and to try try and to try alas on the grass alas the pigeon on the grass the pigeon on the grass and alas.

They might be very well very well very well they might be they might be very well they might be very well very well they might be.

Let Lucy Lily Lily Lucy let Lucy Lucy Lily Lily Lily Lily Lily let Lily Lucy Lucy let Lily. Let Lucy Lily. ${ }^{10}$

La différence qu'établit Roland Barthes, reprenant l'opposition jakobsonienne de la Métaphore et de la Métonymie, est susceptible d'éclairer la cause du travail poétique :

La Métaphore soutient tout discours qui pose la question; "Qu'est-ce que c'est? Qu'est-ce que cela veut dire?»; c'est la question même de tout Essai. La Métonymie, au contraire, pose une autre question: «De quoi ceci, que j'énonce, peut-il être suivi ? Que peut engendrer l'épisode que je raconte?»; c'est la question du Roman. Jakobson rappelait l'expérience menée dans une classe d'enfants ; à qui l'on demandait de réagir au mot « hutte »; les uns répondaient que la hutte est une petite cabane (métaphore), les autres qu'elle avait brûlé (métonymie) [...]. ${ }^{11}$

La métaphore conçue traditionnellement comme la marque du poétique, censée selon Jean Cohen venir " réduire l'écart créé par l'impertinence $~^{12}$, se trouve détrônée au profit d'une poétique du bord-à-bord hantée par la passion du contact, la hantise de la disjonction. À cet égard, la tendance de l'écriture steinienne à composer des « Novellettes » faussement narratives n'est sans doute pas indifférente. La question de la métonymie telle que la formule Roland Barthes pose la question du prochain, de l'à-venir. L'effectuation de l'à-venir du texte est ici une préoccupation cardinale. Notre hypothèse est que la poétique steinienne tend à l'élaboration d'un procès, à la mise au point d'un procédé d'engendrement de la parole. Comment continuer à écrire ? Le poème commence in medias res ou bien s'ouvre de la manière la plus conventionnelle qui soit par une déclinaison d'identité qui peut au passage prendre un tour nonsensique. Ainsi le premier chapitre d'Ida :

There was a baby born named Ida. Its mother held it with her hands to keep Ida from being born but when the time came Ida came. And as Ida came, with her came her twin, so there was Ida-Ida. ${ }^{13}$

Gertrude Stein a l'intuition que le poème ne commence pas ; il a toujours déjà commencé. Le lecteur est bien en peine de dire ce qui justifie qu'un texte interminable comme celui 
de The Making of Americans prenne fin. L'arbitraire est une manière pour les textes de ne commencer ni de finir. Ceux-ci sont comparables en tous points avec le corps au sujet duquel Roland Barthes fait remarquer à propos de Bataille : « le corps ne commence nulle part, il est n'importe où ». Ce «n'importe où » prend chez Stein la forme du « milieu ». Le milieu ne se confond pas avec le centre ; le milieu est un espace homogène, interne, protégé - tout sauf un cadre. La langue est ce milieu, non-lieu par excellence, n'importe où. L'important est que ça continue à s'écrire.

Il n'est pas étonnant que le paragraphe soit distingué par Gertrude Stein comme topos de l'œuvre au détour d'une théorie qui, pour être idiosyncrasique, n'en est pas moins éclairante. Le lieu où faire l'expérience du milieu de la langue est justement le paragraphe. On peut ainsi lire dans « Poetry and Grammar » :

Sentences and paragraphs. Sentences are not emotional but paragraphs are. I can say that as often as I like and it always remains as it is, something that is.

I said I found this out first in listening to Basket my dog drinking. And anybody listening to any dog's drinking will see what I mean.

When I wrote The Making of Americans I tried to break down this essential combination by making enormously long sentences that would be as long as the longest paragraph and so to see if there was really and truly this essential difference between paragraphs and sentences, if one went far enough with this thing with making the sentences long enough to be as long as any paragraph and so producing in them the balance of a paragraph not the balance of a sentence, because of course the balance of a paragraph is not the same balance as the balance of a sentence.

It is only necessary to read anything in order to know that. I say if I succeeded in making my sentences so long that they held within themselves the balance of both sentences and paragraphs, what was the result.

I did in some sentences in The Making of Americans succeed in doing this thing in creating a balance that was neither the balance of a sentence nor the balance of a paragraph and in doing so I felt dimly that I had done something that was not leading to anything because after all you should not lose two things in order to have one thing because in doing so you make writing just much less varied.

That is one thing about what I did. There is also another thing and that was a very important thing, in doing this in achieving something that had neither the balance of a sentence nor the balance of a paragraph but balance a new balance that had to do with a sense of movement of time included in a given space which as I have already said is a definitely American thing. ${ }^{14}$

La différence qu'établit Gertrude Stein ne doit pas être prise à la légère: malgré l'arbitraire dont elle semble procéder, elle révèle le régime de l'écriture fondé sur la digression métonymique à l'infini en même temps qu'elle met l'accent sur l'une de ses découvertes : le paragraphe comme chambre d'échos. Jean-François Lyotard comprend la distinction steinienne de la sorte : «Sur le paragraphe : il sépare ce qu'il unit. Ce mot grec signifie ce qui est écrit à côté. Paragramma est une clause additionnelle à une loi ou à un contrat. Paragraphe une exception opposée par le défenseur à la recevabilité d'une plainte. Paragraphein; ajouter une clause, en particulier frauduleusement (LiddellScott) »" Et l'auteur du Différend d'ajouter: "Dans le texte de Stein, une phrase est une fois, un événement, elle arrive. L'angoisse que ça ne recommence pas, que l'être s'arrête, distende les paragraphes $»^{16}$.

Pourquoi le paragraphe serait-il plus porteur d'affect que la phrase ? Gertrude Stein, en poète syntaxier qu'elle est, n'ignore pas que la phrase, quoiqu'il en soit des transgressions sur lesquelles elle est bâtie, demeure une entité close, constituée d'une série finie de 
relations entre des entités fonctionnelles. Le paragraphe quant à lui n'est pas une entité grammaticalement déterminée. N'étant rien dans le système de la langue, il se prête aux investissements en tous genres. Chambre de résonance, il est un non-lieu grammatical tout entier dédié au travail du signifiant et au régime de la signifiance, lieu d'un possible déchaînement syntaxique, du déchaînement de signifiant, de son avènement sonore. Il est l'occasion de faire l'expérience littérale de l'à-côté du sens. Le paragraphe est le lieu d'une digression éminemment transgressive, puisque celle-ci sonne le glas de la phrase. Il suffit de considérer les phrases-paragraphes que compose Gertrude Stein pour se convaincre de sa puissance de dissolution syntaxique. Le paragraphe en tant qu'unité éclipse la page qu'il lui arrive d'occuper intégralement et pourquoi pas la phrase avec laquelle il coïncide quelquefois. Il est tout sauf une sommation, une addition de phrases. Il donne accès à une dimension inouïe de la langue, la musique. Celle-ci n'est pas audible au niveau de la phrase qui reste prisonnière des relations paradigmatiques qu'elle instaure. Le paragraphe ouvre ainsi sur un espace inconnu et illimité, celui-là même dont parle William Gass à propos de Gertrude Stein, car le paragraphe est le lieu d'un déracinement, de la fin d'un ordre, de sa mise à plat, sa mise en séries non hiérarchiques :

Hoist up a hill. And with certain exceptions modern American writing has been overwhelmed by space: rootlessness, we often say, that's our illness, and we are right; we're sick of changing house, of moving, of cutting loose, of living in vans and riding cycles, of using up and getting on (that's how we age), until sometimes one feels there's nothing but geography in this country, and certainly a geographical history is the only kind it can significantly have; [...]. ${ }^{17}$

Le tropisme métonymique, en même temps qu'il fait dévier le poétique de son axe, fait dérailler la langue, déporte son sujet. Désaxée, l'écriture steinienne rappelle la description qu'un aliéniste donnait dans son Traité clinique des maladies mentales (1886) :

Cette coordination dans la fuite des idées consiste dans une succession d'idées qui ne sont pas amenées primitivement par l'état expansif, mais qui se produisent par suite des associations logiques des pensées et des mots : une idée entraîne toutes les associations possibles... les mots nouveaux et les réminiscences en appellent d'autres, d'une façon particulière à chaque individu. [...] En même temps que les liens logiques s'affaiblissent, les idées du maniaque se relient de plus en plus, et enfin exclusivement, d'après leur forme extérieure, c'est-à-dire d'après la ressemblance du son, d'après l'assonance... le son est tout, le sens n'importe pas... puis ce sont les assonances des syllabes qui sont recherchées ; enfin, au degré le plus bas, viennent des sons inarticulés sifflements, grognements, cris d'animaux). ${ }^{18}$

La poésie de The Making of Americans ne relève pas de la simple graphorée maniaque. Gertrude Stein n'est pas Louis Wolfson, auteur schizophrène du Schizo et les langues ${ }^{19}$ : la pratique poétique de Gertrude Stein n'est pas réductible à un " procédé », pour reprendre le terme dont use Gilles Deleuze pour départir le même Wolfson de Raymond Roussel ${ }^{20}$. La différence capitale réside selon l'auteur de Critique et clinique dans ce qu'il appelle " des visions grandioses » : « Purs événements qui se jouent dans le langage, et qui débordent aussi bien les conditions de leur apparition que les circonstances de leur effectuation, comme une musique excède la circonstance où on la joue et l'exécution qu'on en fait ${ }^{21}$. Il suffit de considérer un vers pour s'en convaincre : «Rose is a rose is a rose is a rose $»^{22}$. Le rosaire le plus célèbre de la littérature américaine, tiré de Sacred Emily et repris dans The World is Round ${ }^{23}$, ne se résout pas dans la seule répétition de mots. La rose devient guirlande, celle-là même dont on couronnait la Vierge - sens premier et étymologique du rosaire. Punctum de l'œuvre, le rosaire est une forme poétique steinienne avant la lettre 
puisqu'il consistait traditionnellement dans la récitation de quinze dizaines d'Ave précédés chacune d'un Pater en l'honneur du mystère de l'incarnation.

Le déficit de marge que présente le texte de The Making of Americans est plus qu'un symptôme ou une curiosité : c'est un indice, il fait index vers le bord vers lequel tend l'écriture. Le bord visé par cette poétique de la métonymie est le bord de la langue, la langue étant comme bord, comme faisant bord avec le réel. La nature même du bord s'en trouve dévoilée dans son ambiguïté constitutive: lieu d'un contact, le bord est aussi le lieu d'une scansion, d'un basculement.

\section{NOTES}

1. Serpent de mer de la première partie de l'œuvre steinienne, The Making of Americans, commencé en 1903, achevé en 1908, ne trouva d'éditeur qu'en 1925.

2. Cuvres complètes, Paris : Gallimard, 1945, 477.

3. The Autobiography of Alice B. Toklas, Stein Writings 1903-1932, New York: The Library of America, 1998, 729-730.

4. La Cruauté mélancolique, Paris : Flammarion, 1998, 99.

5. How Writing is Written, Robert Bartlett Haas (ed.), Los Angeles: Black Sparrow Press, 1974, 153.

6. "Poetry and Grammar", Writings 1932-1946, New York: The Library of America, 1998, 318.

7. Ibid., 318. Nous soulignons.

8. Article paru dans L'Euvre, 5 février 1935 et signé H.S.

9. Paris : Flammarion, 1966, 92.

10. Writings 1903-1932, op.cit., 637.

11. "Longtemps je me suis couché de bonne heure »; Le Bruissement de la langue, Paris: Seuil, 1984, 335.

12. Jean Cohen, op.cit., 114.

13. Writings 1932-1946, op.cit., 611.

14. Ibid., 322.

15. Le Différend, Paris : Minuit, 1984, 104.

16. Ibid., 106.

17. “Gertrude Stein, Geographer: I", New York Review of Books 20.7 (May 3, 1973).

18. Cité par Louis Sciara, Le Discours Psychanalytique, « La Ponctuation », octobre 1997, 141.

19. Paris : Gallimard, 1970.

20. Critique et clinique, Paris : Minuit, 1993, 21.

21. Ibid., 21-22.

22. Writings 1903-1932, op.cit., 395.

23. Writings 1932-1946, op.cit., 537. 
INDEX

oeuvrecitee Making of the Americans (The) 